\title{
Periodic solutions for some phi-Laplacian and reflection equations
}

\author{
Alberto Cabada* and Fernando Adrián Fernández Tojo
}

\author{
"Correspondence: \\ alberto.cabada@usc.es \\ Departamento de Análise \\ Matemática, Facultade de \\ Matemáticas, Universidade de \\ Santiago de Compostela, Santiago \\ de Compostela, Spain
}

\begin{abstract}
This work is devoted to the study of the existence and periodicity of solutions of initial differential problems, paying special attention to the explicit computation of the period. These problems are also connected with some particular initial and boundary value problems with reflection, which allows us to prove existence of solutions of the latter using the existence of the former.
\end{abstract}

Keywords: equations with involutions; equations with reflection; $\phi$-Laplacian; periodic solutions

\section{Introduction}

The idea behind this paper appeared in another work of the authors [1] where the following lemmas were proved.

Definition 1.1 If $A \subset \mathbb{R}$, a function $\varphi: A \rightarrow A$ such that $\varphi \neq \operatorname{Id}$ and $\varphi \circ \varphi=\mathrm{Id}$ is called an involution.

Let us consider the problems

$$
x^{\prime}(t)=f(x(\varphi(t))), \quad x(c)=x_{c},
$$

and

$$
x^{\prime \prime}(t)=f^{\prime}\left(f^{-1}\left(x^{\prime}(t)\right)\right) f(x(t)) \varphi^{\prime}(t), \quad x(c)=x_{c}, \quad x^{\prime}(c)=f\left(x_{c}\right) .
$$

Lemma 1.1 ([1], Lemma 2.1) Let $(a, b) \subset \mathbb{R}$ and let $f: \mathbb{R} \rightarrow \mathbb{R}$ be a diffeomorphism. Let $\varphi \in \mathcal{C}^{1}((a, b))$ be an involution. Let $c$ be a fixed point of $\varphi$. Then $x$ is a solution of the first order differential equation with involution (1) if and only if $x$ is a solution of the second order ordinary differential equation (2).

Furthermore, a version of Lemma 1.1 can be proved for the case with periodic boundary value conditions.

Let us consider the equations

$$
x^{\prime}(t)=f(x(\varphi(t))), \quad x(a)=x(b),
$$

(c) 2016 Cabada and Fernández Tojo. This article is distributed under the terms of the Creative Commons Attribution 4.0 International License (http://creativecommons.org/licenses/by/4.0/), which permits unrestricted use, distribution, and reproduction in any medium, provided you give appropriate credit to the original author(s) and the source, provide a link to the Creative Commons license, and indicate if changes were made. 
and

$$
x^{\prime \prime}(t)=f^{\prime}\left(f^{-1}\left(x^{\prime}(t)\right)\right) f(x(t)) \varphi^{\prime}(t), \quad x(a)=x(b)=f^{-1}\left(x^{\prime}(a)\right) .
$$

Lemma 1.2 ([1], Lemma 2.2) Let $[a, b] \subset \mathbb{R}$ and let $f: \mathbb{R} \rightarrow \mathbb{R}$ be a diffeomorphism. Let $\varphi \in \mathcal{C}^{1}([a, b])$ be an involution such that $\varphi([a, b])=[a, b]$. Then $x$ is a solution of the first order differential equation with involution (3) if and only if $x$ is a solution of the second order ordinary differential equation (4).

Remark 1.1 Although not stated in [1], it is important to notice that, taking into account Theorem 9.3 in [2] and Theorem 6.93 in [3], the proofs of Lemmas 1.1 and 1.2 are still valid if we weaken the regularity hypothesis on $f$ and $f^{-1}$ to $f$ and $f^{-1}$ being absolutely continuous and $f$ locally Lipschitz.

Linear problems with involutions, similar to problem (1), have also been studied in $[1,4$, 5]. Observe that from problem (4) we have

$$
\begin{aligned}
0 & =\frac{x^{\prime \prime}(t)}{f^{\prime}\left(f^{-1}\left(x^{\prime}(t)\right)\right)}-f(x(t)) \varphi^{\prime}(t)=\left(f^{-1}\right)^{\prime}\left(x^{\prime}(t)\right) x^{\prime \prime}(t)-f(x(t)) \varphi^{\prime}(t) \\
& =\left(f^{-1} \circ x^{\prime}\right)^{\prime}(t)-f(x(t)) \varphi^{\prime}(t) .
\end{aligned}
$$

So, clearly, problem (4) is equivalent to the problem

$$
\left(f^{-1} \circ x^{\prime}\right)^{\prime}(t)-\varphi^{\prime}(t) f(x(t))=0, \quad x(a)=x(b), \quad x^{\prime}(a)=f(x(a)) .
$$

This involves the $f^{-1}$-Laplacian $\left(f^{-1} \circ x^{\prime}\right)^{\prime}$, although, contrary to most literature, the other term in the equation does not involve $f^{-1}$ but $f$. As we will see, this is not more than a further generalization in the line of the $p$ - $q$-Laplacian.

Problems concerning the $\phi$-Laplacian (or, particularly, the $p$-Laplacian) have been studied extensively in recent literature. Drábek, Manásevich and others study the eigenvalues of problems with the $p$-Laplacian in [6-10] using variational methods. The existence of positive solutions is treated in [11], the existence of an exact number of solutions in [12] and topological existence results can be found in [13]. Anti-maximum principles and sign properties of the solutions are studied in $[14,15]$. In [16] one studies a variant of the $p$ Laplacian equation with an approach based on variational methods, in [17] one studies the eigenvalues of the Dirichlet problem, and in [18] one finds some oscillation criteria for equations with the $p$-Laplacian.

The $\phi$-Laplacian is studied from different points of view in several papers, e.g. [19-25]. Actually, if we consider the problem with the $f^{-1}$-Laplacian

$$
\left(f^{-1} \circ x_{c}^{\prime}\right)^{\prime}(t)+f\left(x_{c}(t)\right)=0, \quad x_{c}(a)=c, \quad x_{c}^{\prime}(a)=f(c),
$$

and we assume there exist $\bar{c}_{1}, \bar{c}_{2} \in \mathbb{R}, \bar{c}_{1}<\bar{c}_{2}$, such that a unique solution of problem (6) exists for every $c \in\left[\bar{c}_{1}, \bar{c}_{2}\right]$ and $\left(x_{\bar{c}_{1}}(b)-\bar{c}_{1}\right)\left(x_{\bar{c}_{2}}(b)-\bar{c}_{2}\right)<0$, then problem (4) must have at least a solution due to the continuity of $x_{c}$ on $c$ and Bolzano's theorem. For this reason we will be interested in studying the properties of problem (6) and its solutions in this paper. In the sections to come we study this problem and more general versions of it. 
In the following section we will study the existence, uniqueness and periodicity of solutions of problem (7) and in Section 3 we will apply these results to the case of problems with reflection.

\section{General solutions}

First, we write in a general way the solutions of equations involving the $g$ - $f$-Laplacian.

Let $\tau_{i}, \sigma_{i} \in[-\infty, \infty], i=1, \ldots, 4, \tau_{1}<\tau_{2}, \sigma_{1}<\sigma_{2}, \tau_{3}<\tau_{4}, \sigma_{3}<\sigma_{4}$. Let $f:\left(\tau_{1}, \tau_{2}\right) \rightarrow\left(\sigma_{1}, \sigma_{2}\right)$ and $g:\left(\tau_{3}, \tau_{4}\right) \rightarrow\left(\sigma_{3}, \sigma_{4}\right)$ be invertible functions such that $f$ and $g^{-1}$ are continuous. Assume there is $s_{0} \in\left(\tau_{1}, \tau_{2}\right)$ such that $f\left(s_{0}\right)=0$ and define $F(t):=\int_{s_{0}}^{t} f(s) \mathrm{d} s$. Observe that $F$ is 0 at $s_{0}$ and of constant sign everywhere else. The following lemma is a straightforward application of the properties of the integral.

Lemma 2.1 If $f$ is continuous, invertible and increasing (decreasing) then $\left.F_{-} \equiv F\right|_{\left(-\infty, s_{0}\right]}$ is strictly decreasing (increasing) and $\left.F_{+} \equiv F\right|_{\left[s_{0},+\infty\right.}$ is strictly increasing (decreasing). Furthermore, if $\tau_{1}=-\infty, F(-\infty)=+\infty(-\infty)$ and if $\tau_{2}=+\infty, F(+\infty)=+\infty(-\infty)$.

All the same, define $G(t):=\int_{g^{-1}(\{0\})}^{t} g^{-1}(s) \mathrm{d} s$ and consider the problem

$$
\left(g \circ x^{\prime}\right)^{\prime}(t)+f(x(t))=0, \quad \text { a.e. } t \in \mathbb{R} ; \quad x(a)=c_{1}, \quad x^{\prime}(a)=c_{2},
$$

for some fixed $c_{1}, c_{2} \in \mathbb{R}$.

Definition 2.1 A solution $x$ of problem (7) will be $x \in \mathcal{C}^{1}(I)$, such that $g \circ x^{\prime}$ is absolutely continuous on $I$, where $I$ is an open interval with $a \in I$. The solution must further satisfy the requirement that the equation in problem (7) holds a.e. and the initial conditions are satisfied as well.

Theorem 2.2 Let $f:\left(\tau_{1}, \tau_{2}\right) \rightarrow\left(\sigma_{1}, \sigma_{2}\right)$ and $g:\left(\tau_{3}, \tau_{4}\right) \rightarrow\left(\sigma_{3}, \sigma_{4}\right)$ be invertible functions such that $f$ and $g^{-1}$ are continuous and assume $0 \in\left(\tau_{1}, \tau_{2}\right) \cap\left(\tau_{3}, \tau_{4}\right), f(0)=0, g(0)=0$, $f$ and $g$ increasing, $F\left(c_{1}\right)+G\left(g\left(c_{2}\right)\right)<\min \left\{G\left(\sigma_{3}\right), G\left(\sigma_{4}\right)\right\}$. Then there exists a unique local solution of problem (7).

Furthermore, if $F\left(c_{1}\right)+G\left(g\left(c_{2}\right)\right)<\min \left\{F\left(\tau_{1}\right), F\left(\tau_{2}\right)\right\}$, then such solution is defined on the whole real line and is periodic of smallest period

$$
\begin{aligned}
T:= & \int_{F_{-}^{-1}\left(G\left(g\left(c_{2}\right)\right)+F\left(c_{1}\right)\right)}^{F_{+}^{-1}\left(G\left(g\left(c_{2}\right)\right)+F\left(c_{1}\right)\right)}\left[\frac{1}{\left(g^{-1} \circ G_{+}^{-1}\right)\left(G\left(g\left(c_{2}\right)\right)+F\left(c_{1}\right)-F(r)\right)}\right. \\
& \left.-\frac{1}{\left(g^{-1} \circ G_{-}^{-1}\right)\left(G\left(g\left(c_{2}\right)\right)+F\left(c_{1}\right)-F(r)\right)}\right] \mathrm{d} r .
\end{aligned}
$$

Proof For the first part of the theorem and without loss of generality, we will prove the existence of solution in an interval of the kind $[a, a+\delta), \delta \in \mathbb{R}^{+}$. The proof would be analogous for an interval of the kind $(a-\delta, a]$.

Let $y(t)=g\left(x^{\prime}(t)\right)$. Then problem (7) is equivalent to

$$
x^{\prime}(t)=g^{-1}(y(t)), \quad y^{\prime}(t)=-f(x(t)), \quad t \in \mathbb{R}, \quad x(a)=c_{1}, \quad y(a)=g\left(c_{2}\right) .
$$


Hence,

$$
f(x(t)) x^{\prime}(t)+g^{-1}(y(t)) y^{\prime}(t)=0, \quad t \in \mathbb{R}
$$

so, integrating both sides from $a$ to $t$,

$$
F(x(t))+G(y(t))=k, \quad t \in \mathbb{R},
$$

where $k=F\left(c_{1}\right)+G\left(g\left(c_{2}\right)\right)$. That is, undoing the change of variables,

$$
G\left(g\left(x^{\prime}(t)\right)\right)=G\left(g\left(c_{2}\right)\right)+F\left(c_{1}\right)-F(x(t)), \quad t \in \mathbb{R} .
$$

If $c_{1}=c_{2}=0$ it is clear that the only possible solution is $x \equiv 0$ for, in that case, $G\left(g\left(x^{\prime}(t)\right)\right)+$ $F(x(t))=0$ and, since $G$ and $F$ are nonnegative and increasing, $x^{\prime}(t)=x(t)=0$ for $t \in \mathbb{R}$. Assume, without loss of generality, that $c_{2}$ is nonnegative and $c_{1}$ negative (the other cases are similar). If $c_{2}=0$ then, integrating (7),

$$
g \circ x^{\prime}(t)=-\int_{a}^{t} f(x(s)) \mathrm{d} s
$$

which implies $x^{\prime}$ is positive in some interval $[a, a+\delta)$.

If $c_{2}$ is positive, then $x^{\prime}$ has to be positive at least in some neighborhood of $a$, so, in a right neighborhood of $a$, we can solve for $g \circ x^{\prime}$ in (9) as

$$
g \circ x^{\prime}(t)=G_{+}^{-1}\left(F\left(c_{1}\right)-F(x(t))+G\left(g\left(c_{2}\right)\right)\right) .
$$

In order to solve for $x^{\prime}$ in (10), we need $F\left(c_{1}\right)+G\left(g\left(c_{2}\right)\right)<G\left(\sigma_{4}\right)$. Then

$$
x^{\prime}(t)=\left(g^{-1} \circ G_{+}^{-1}\right)\left(F\left(c_{1}\right)-F(x(t))+G\left(g\left(c_{2}\right)\right)\right) .
$$

Integrating between $a$ and $t$,

$$
t=\int_{a}^{t} \frac{x^{\prime}(s)}{\left(g^{-1} \circ G_{+}^{-1}\right)\left(F\left(c_{1}\right)-F(x(s))+G\left(g\left(c_{2}\right)\right)\right)} \mathrm{d} s+a=H_{+}(x(t)),
$$

where

$$
H_{+}(r):=\int_{c_{1}}^{r} \frac{1}{\left(g^{-1} \circ G_{+}^{-1}\right)\left(F\left(c_{1}\right)-F(s)+G\left(g\left(c_{2}\right)\right)\right)} \mathrm{d} s+a .
$$

$H_{+}$is strictly increasing in its domain due to the positivity of the denominator in the integrand. Hence, for $t$ sufficiently close to $a$,

$$
x(t)=H_{+}^{-1}(t)
$$

Therefore, a solution of problem (7) exists and is unique (by construction) on an interval $[a, a+\delta)$. 
If we assume $F\left(c_{1}\right)+G\left(g\left(c_{2}\right)\right)<\min \left\{F\left(\tau_{1}\right), F\left(\tau_{2}\right)\right\}, c_{2}>0$ (the case $c_{2}=0$ is similar), $H_{+}$is well defined on

$$
I:=\left(F_{-}^{-1}\left(F\left(c_{1}\right)+G\left(g\left(c_{2}\right)\right)\right), F_{+}^{-1}\left(F\left(c_{1}\right)+G\left(g\left(c_{2}\right)\right)\right)\right) .
$$

Now, we study the range of $H_{+}$.

$g\left(x^{\prime}(t)\right)$ is positive as long as $x^{\prime}(t)$ is positive. Hence, consider

$$
t_{0}:=\sup \left\{t \in[a,+\infty): x^{\prime}(s)>0 \text { for a.e. } s \in[a, t)\right\} \in[a,+\infty]
$$

$G$ is positive on nonzero values, so equation (9) implies that

$$
F(x(t))<G\left(g\left(c_{2}\right)\right)+F\left(c_{1}\right)
$$

for all $t \in\left(a, t_{0}\right)$.

Assume $t_{0}=+\infty$. Now, $x^{\prime}(t)>0$ a.e. in $[a,+\infty)$ so there exists

$$
x(+\infty) \in\left(c_{1}, F_{+}^{-1}\left(G\left(g\left(c_{2}\right)\right)+F\left(c_{1}\right)\right)\right] .
$$

On the other hand, since $x$ is increasing in $[a,+\infty)$ and $c_{1}<0$, by equation (11) we see that $x^{\prime}$ is increasing as long as $x$ is negative. This means that, eventually (in finite time), $x$ will be positive and therefore, $x^{\prime}$ is decreasing in $[\tilde{a},+\infty)$ for $\tilde{a}$ big enough, so there exists $x^{\prime}(+\infty) \geq 0$. If we assume $x^{\prime}(+\infty)=\epsilon>0$, this implies that $x(+\infty)=+\infty$, for there would exist $M \in \mathbb{R}$ such that $x^{\prime}(t)>\epsilon / 2$ for every $t \geq M$, so $x^{\prime}(+\infty)=0$. Taking the limit $t \rightarrow+\infty$ in equation (9), $x(+\infty)=F_{+}^{-1}\left(G\left(g\left(c_{2}\right)\right)+F\left(c_{1}\right)\right)$.

Now, take $\epsilon \in(0, f(x(+\infty)))$. Since $g \circ x^{\prime}(+\infty)=0$ and $g \circ x^{\prime}$ is continuous and decreasing in $[\tilde{a},+\infty)$, there exists $M \in \mathbb{R}^{+}$such that $\left|g\left(x^{\prime}\left(M_{2}\right)\right)-g\left(x^{\prime}\left(M_{1}\right)\right)\right|<\epsilon$ for every $M_{1}, M_{2}>M$. Since $f$ is continuous, there exists $\tilde{M}>M$ such that $f\left(x\left(M_{3}\right)\right)>\epsilon$ for every $M_{3}>\tilde{M}$. Take $M_{3}$ in such a way. Then, integrating equation (7) between $M_{3}$ and $M_{3}+1$,

$$
\left(g \circ x^{\prime}\right)\left(M_{3}+1\right)-\left(g \circ x^{\prime}\right)\left(M_{3}\right)=\int_{M_{3}}^{M_{3}+1} f(x(s)) \mathrm{d} s>\epsilon,
$$

a contradiction. Therefore, $t_{0} \in \mathbb{R}$.

Observe that $x^{\prime}\left(t_{0}\right)=0$, so $x$ attains its maximum at $t_{0}$ and $x\left(t_{0}\right)=F_{+}^{-1}\left(G\left(g\left(c_{2}\right)\right)+F\left(c_{1}\right)\right)$ by equation (9), that is, $x\left(t_{0}\right)=\sup I$. In order for this value to be well defined it is necessary that $G\left(g\left(c_{2}\right)\right)+F\left(c_{1}\right) \leq F\left(\tau_{2}\right)$.

Now, we see that $H_{+}$is well defined at $\sup I$ (assuming it is defined continuous at that point). Indeed,

$$
t_{0}=\lim _{t \rightarrow t_{0}} H_{+}(x(t))=H_{+}\left(F_{+}^{-1}\left(G\left(g\left(c_{2}\right)\right)+F\left(c_{1}\right)\right)\right) .
$$

We prove now that there is a neighborhood $\left(t_{0}, t_{0}+\epsilon\right)$ where $x^{\prime}$ is negative, which means that we can take

$$
t_{1}:=\sup \left\{t \in\left[t_{0},+\infty\right): x^{\prime}(s)<0 \text { for a.e. } s \in\left[t_{0}, t\right)\right\}
$$


Fix $\xi$ such that $0<\xi<f\left(x\left(t_{0}\right)\right)$ and take $\epsilon$ such that $f(x(t))>\xi$ in $\left(t_{0}, t_{0}+\epsilon\right)$. Take $t \in$ $\left(t_{0}, t_{0}+\epsilon\right)$, then, integrating equation (7) between $t_{0}$ and $t$,

$$
g\left(x^{\prime}(t)\right)=-\int_{t_{0}}^{t} f(x(s)) \mathrm{d} s<-\xi\left(t-t_{0}\right)<0
$$

We deduce that $t_{1}<+\infty$ by the same kind of reasoning we used to prove $t_{0}<+\infty$. Observe that $x^{\prime}\left(t_{1}\right)=0$ and $x\left(t_{1}\right)=F_{-}^{-1}\left(G\left(g\left(c_{2}\right)\right)+F\left(c_{1}\right)\right)$. This last equality comes from evaluating equation (9) at $t_{1}$ and Rolle's theorem as we show now: the other possibility would be $x\left(t_{1}\right)=$ $F_{+}^{-1}\left(G\left(g\left(c_{2}\right)\right)+F\left(c_{1}\right)\right)$. Observe that, by equation (11), $x^{\prime}$ is continuous, so $x \in \mathcal{C}^{1}\left(\left[a, t_{1}\right)\right)$. Since $x\left(t_{0}\right)=x\left(t_{1}\right)$, there would exist $\tilde{t} \in\left(t_{0}, t_{1}\right)$ such that $x^{\prime}(\tilde{t})=0$, a contradiction.

Now, we see that $x^{\prime}(t)=\left(g^{-1} \circ G_{-}^{-1}\right)\left(G\left(g\left(c_{2}\right)\right)+F\left(c_{1}\right)-F(x(t))\right)$, that is,

$$
1=x^{\prime}(t) /\left(g^{-1} \circ G_{-}^{-1}\right)\left(G\left(g\left(c_{2}\right)\right)+F\left(c_{1}\right)-F(x(t))\right) .
$$

Thus,

$$
\begin{aligned}
t_{1}-t_{0} & =\int_{t_{0}}^{t_{1}} \frac{x^{\prime}(s) \mathrm{d} s}{\left(g^{-1} \circ G_{-}^{-1}\right)\left(G\left(g\left(c_{2}\right)\right)+F\left(c_{1}\right)-F(x(s))\right)} \\
& =\int_{F_{+}^{-1}\left(G\left(g\left(c_{2}\right)\right)+F\left(c_{1}\right)\right)}^{F_{-}^{-1}\left(G\left(g\left(c_{2}\right)\right)+F\left(c_{1}\right)\right)} \frac{\mathrm{d} r}{\left(g^{-1} \circ G_{-}^{-1}\right)\left(G\left(g\left(c_{2}\right)\right)+F\left(c_{1}\right)-F(r)\right)} .
\end{aligned}
$$

If we define

$$
H_{-}(s):=\int_{F_{+}^{-1}\left(G\left(g\left(c_{2}\right)\right)+F\left(c_{1}\right)\right)}^{s} \frac{\mathrm{d} r}{\left(g^{-1} \circ G_{-}^{-1}\right)\left(G\left(g\left(c_{2}\right)\right)+F\left(c_{1}\right)-F(r)\right)}+t_{0},
$$

$H_{-}$is strictly decreasing in its domain and $x(t)=H_{-}^{-1}(t)$ for $t \in\left[t_{0}, t_{1}\right]$.

We can again deduce that

$$
t_{2}:=\sup \left\{t \in\left[t_{1},+\infty\right): x^{\prime}(s)>0 \text { for a.e. } s \in\left[t_{1}, t\right)\right\}<+\infty
$$

Using the positivity and growth conditions of the functions involved, it is easy to check that $x\left(t_{1}\right)=F_{-}^{-1}\left(G\left(g\left(c_{2}\right)\right)+F\left(c_{1}\right)\right)<c_{1}<F_{+}^{-1}\left(G\left(g\left(c_{2}\right)\right)+F\left(c_{1}\right)\right)=x\left(t_{2}\right)$, so there exists a unique $b \in\left(t_{1}, t_{2}\right)$ such that $x(b)=c_{1}$. Now,

$$
\begin{aligned}
b-t_{1} & =\int_{t_{1}}^{b} \frac{x^{\prime}(s) \mathrm{d} s}{\left(g^{-1} \circ G_{+}^{-1}\right)\left(G\left(g\left(c_{2}\right)\right)+F\left(c_{1}\right)-F(x(s))\right)} \\
& =\int_{F_{-}^{-1}\left(G\left(g\left(c_{2}\right)\right)+F\left(c_{1}\right)\right)}^{c_{1}} \frac{\mathrm{d} r}{\left(g^{-1} \circ G_{+}^{-1}\right)\left(G\left(g\left(c_{2}\right)\right)+F\left(c_{1}\right)-F(r)\right)} .
\end{aligned}
$$

Defining $T:=b-a$ and extending $x$ periodically in the following way (we have $x$ already defined in $[a, a+T])$ :

$$
x(t)=x\left(t-\left\lfloor\frac{t-a}{T}\right\rfloor T\right),
$$

where $\lfloor t\rfloor:=\sup \{k \in \mathbb{Z}: k \leq t\}$, it is easy to check that $x$, extended in such a way, is a global periodic solution of problem (7). 
Take $z(t):=x(t-T), t \in \mathbb{R}$, we show that $z$ is a solution of the problem in $[a+T, a+2 T]$.

$$
0=\left(g \circ x^{\prime}\right)^{\prime}(t)+f(x(t))=\left(g \circ z^{\prime}\right)^{\prime}(t+T)+f(z(t+T)) \quad \text { for a.e. } t \in \mathbb{R} \text {. }
$$

This is equivalent to

$$
\left(g \circ z^{\prime}\right)^{\prime}(t)+f(z(t))=0 \quad \text { for a.e. } t \in \mathbb{R} \text {. }
$$

Also,

$$
\begin{aligned}
& z(a+T)=x(a)=c_{1}, \\
& z^{\prime}(a+T)=x^{\prime}(a)=c_{2} .
\end{aligned}
$$

Remark 2.1 A similar argument can be given for the case $f$ and $g$ have different growth type (e.g. $f$ increasing and $g$ decreasing), but taking the negative branch of the inverse function $G^{-1}$ in (11).

Remark 2.2 In the hypotheses of Theorem 2.2, if instead of $g(0)=f(0)=0$ we see that $g\left(s_{0}\right)=f\left(s_{0}\right)=0$, define $\tilde{f}(x):=f\left(x+s_{0}\right), \tilde{g}(x):=g\left(x+s_{0}\right)$. Then $\tilde{f}(0)=\tilde{g}(0)=0$ and problem (7) is equivalent to

$$
\left(\tilde{g} \circ v^{\prime}\right)^{\prime}(t)+\tilde{f}(v(t))=0, \quad v(a)=c_{1}-s_{0}, \quad v(a)=c_{2},
$$

with $v(t)=x(t)-s_{0}$. Hence, we can apply Theorem 2.2 to this case.

Remark 2.3 Using the notation of Theorem 2.2, the explicit form of the solution of problem (7) is given by

$$
x(t)= \begin{cases}H_{+}^{-1}\left(t-\left\lfloor\frac{t-a}{T}\right\rfloor T\right), & t \in[a+2 T k, a+(2 k+1) T], k \in \mathbb{Z}, \\ H_{-}^{-1}\left(t-\left\lfloor\frac{t-a}{T}\right\rfloor T\right), & t \in[a+(2 k-1) T, a+2 k T], k \in \mathbb{Z} .\end{cases}
$$

Remark 2.4 Consider the following particular case of problem (7) with $f(0)=0, g(0)=0$, $f$ and $g$ increasing, and the hypothesis for a unique global solution of the following problem satisfied in Theorem 2.2.

$$
\left(g \circ x^{\prime}\right)^{\prime}(t)+f(x(t))=0, \quad x(0)=0, \quad x^{\prime}(0)=1 .
$$

It is clear that, in the case $g(x)=f(x)=x$, the unique solution of problem (12) is $\sin (t)$, which suggests the definition of the $\sin _{g, f}$ function as the unique solution of problem (12) for general $g$ and $f$. Correspondingly,

$$
\arcsin _{g, f}^{+}(r):=H_{+}(r)
$$

This function, defined as such, coincides with the $\arcsin _{p}$ function defined in [26, 27] for the $p$-Laplacian $f(x)=g(x)=|x|^{p-2} x$, the function $\arcsin _{p, q}$ defined in [28-30] for the $p-q$ Laplacian $f(x)=|x|^{q-2} x, g(x)=|x|^{p-2} x$, which first appeared with a slightly different definition in [8], and the hyperbolic version of this function, also in $[29,30]$, which corresponds 
to the case $f(x)=|x|^{q-2} x, g(x)=-|x|^{p-2} x$. [31] derives generalized Jacobian functions in a similar way, defining

$$
\operatorname{arcsn}_{p, q}(t, k):=\int_{0}^{t} \frac{1}{\sqrt[p]{\left(1-s^{q}\right)\left(1-k^{q} s^{q}\right)}} \mathrm{d} s
$$

of which the inverse (see [31], Proposition 3.2) is precisely a solution of

$$
\left(f_{p} \circ x^{\prime}(t)\right)^{\prime}+\frac{q}{p^{*}} f_{q}(x(t))\left(1+k^{q}-2 k^{q}|x(t)|^{q}\right)=0,
$$

where $f_{r}$ is the $r$-Laplacian for $r=p, q$ and $p^{*} p=p^{*}+p$. Observe that this case is also covered by our definition.

In all of the aforementioned work one is interested in the inverse of the $\arcsin _{g, f}$ function, the $\sin _{g, f}$ function, which is extended to the whole real line by symmetry and periodicity. Observe that in our case $f$ and $g$ need not to be odd functions, contrary to the above examples, but we can still give the definition of the $\sin _{g, f}$ function in the whole real line. Also, this lack of symmetry gives rise to a richer set of right inverses of $\sin _{g, f}$, for instance,

$$
\arcsin _{g, f}^{-}(r):=H_{-}(r)
$$

In general, if we have a problem of the kind

$$
\Phi\left(\left(g \circ x^{\prime}\right)^{\prime}, x(t)\right)=0 ; \quad x(0)=0, \quad x^{\prime}(0)=1,
$$

and we know it has a unique solution in a neighborhood of 0 , then we can define $\sin _{g, \Phi}$ as such unique solution and its inverse, in a neighborhood of $0, \arcsin _{g, \Phi}$.

\subsection{A particular case}

Having in mind problem (6), we now consider a particular case of problem (7) for the rest of this section. Assume $f$ is invertible and both $f$ and $f^{-1}$ are continuous. For convenience, assume also that $f$ is increasing and $f(0)=0$. Consider the following problem.

$$
\left(f^{-1} \circ x^{\prime}\right)^{\prime}(t)+\lambda f(x(t))=0, \quad x(a)=c, \quad x^{\prime}(a)=f(c),
$$

where $\lambda \in \mathbb{R}^{+}$.

The following corollary is just the restatement of Theorem 2.2 for this particular case.

Corollary 2.3 Let $f:\left(\tau_{1}, \tau_{2}\right) \rightarrow\left(\sigma_{1}, \sigma_{2}\right)$ be an invertible function such that $f$ is continuous and assume $0 \in\left(\tau_{1}, \tau_{2}\right), f(0)=0$, and $f$ increasing, $\lambda>0,(1+\lambda) F(c)<\min \left\{F\left(\tau_{1}\right), F\left(\tau_{2}\right)\right\}$. Then there exists a unique local solution of problem (13).

Furthermore, if $\left(1+\lambda^{-1}\right) F(c)<\min \left\{F\left(\tau_{1}\right), F\left(\tau_{2}\right)\right\}$, then such solution is defined on $\mathbb{R}$ and is periodic of first period

$$
T:=\int_{F_{-}^{-1}\left(\left(1+\lambda^{-1}\right) F(c)\right)}^{F_{+}^{-1}\left(\left(1+\lambda^{-1}\right) F(c)\right)}\left[\frac{1}{f\left(F_{+}^{-1}((1+\lambda) F(c)-\lambda F(r))\right)}-\frac{1}{f\left(F_{-}^{-1}((1+\lambda) F(c)-\lambda F(r))\right)}\right] \mathrm{d} r .
$$


There are some particular cases where the formula (14) can be simplified.

If $f$ is odd then $F$ is even and, with the change of variables $r=|c| s$, we see that expression (14) becomes

$$
T=\int_{0}^{\frac{F_{+}^{-1}\left(\left(1+\lambda^{-1}\right) F(c)\right)}{|c|}} \frac{4|c| \mathrm{d} r}{f\left(F_{+}^{-1}((1+\lambda) F(c)-\lambda F(|c| r))\right)} .
$$

Also, if we further assume that $f$ is defined in $\mathbb{R}$ and that $f(r t)=h(r) f(t)$ for every $r, t \in \mathbb{R}$ (see Remark 2.5 for a classification of such functions) and some function $h$, then

$$
F(r t)=\int_{0}^{r t} f(s) \mathrm{d} s=\int_{0}^{t} f(r s) r \mathrm{~d} s=r h(r) \int_{0}^{t} f(s) \mathrm{d} s=r h(r) F(t)
$$

so $F$ satisfies the same kind of property for $\tilde{h}(r)=r h(r)$.

Clearly, for $t>0$,

$$
F_{-}^{-1}(\tilde{h}(r) t)=r F_{-}^{-1}(t), \quad F_{+}^{-1}(\tilde{h}(r) t)=r F_{+}^{-1}(t)
$$

Observe that $\tilde{h}(r)=F(r) / F(1)$, and therefore $\left.\tilde{h}\right|_{(-\infty, 0]},\left.\tilde{h}\right|_{[0,+\infty)}$ are invertible. Also, $\tilde{h}_{+}^{-1}(t)=$ $F_{+}^{-1}(t F(1))$ for $t>0$. Hence,

$$
\begin{aligned}
\frac{F_{+}^{-1}\left(\left(1+\lambda^{-1}\right) F(c)\right)}{|c|} & =\frac{F_{+}^{-1}\left(\tilde{h}\left(\tilde{h}_{+}^{-1}\left(1+\lambda^{-1}\right)\right) F(c)\right)}{|c|}=\frac{\tilde{h}_{+}^{-1}\left(1+\lambda^{-1}\right) F_{+}^{-1}(F(c))}{|c|} \\
& =\tilde{h}_{+}^{-1}\left(1+\lambda^{-1}\right)=F_{+}^{-1}\left(\left(1+\lambda^{-1}\right) F(1)\right) .
\end{aligned}
$$

All the same, $F_{-}^{-1}\left(\left(1+\lambda^{-1}\right) F(c)\right) /|c|=-F_{-}^{-1}\left(\left(1+\lambda^{-1}\right) F(1)\right)$.

Also,

$$
\begin{aligned}
f\left(F_{+}^{-1}((1+\lambda) F(c)-\lambda F(|c| r))\right) & =f\left(F_{+}^{-1}((1+\lambda) \tilde{h}(|c|) F(1)-\lambda \tilde{h}(|c|) F(r))\right) \\
& =f\left(F_{+}^{-1}(\tilde{h}(|c|)[(1+\lambda) F(1)-\lambda F(r))]\right) \\
& =f\left(|c| F_{+}^{-1}((1+\lambda) F(1)-\lambda F(r))\right) \\
& =h(|c|) f\left(F_{+}^{-1}((1+\lambda) F(1)-\lambda F(r))\right) \\
& =(f(|c|) / f(1)) f\left(F_{+}^{-1}((1+\lambda) F(1)-\lambda F(r))\right) .
\end{aligned}
$$

With these considerations in mind, we see that we can further reduce expression (14) to

$$
T(c, \lambda)=\frac{4|c| f(1)}{f(|c|)} \int_{0}^{F_{+}^{-1}\left(\left(1+\lambda^{-1}\right) F(1)\right)} \frac{\mathrm{d} r}{f\left(F_{+}^{-1}((1+\lambda) F(1)-\lambda F(r))\right)} .
$$

Example 2.1 Let $f(t):=|t|^{p-2} t, p>1$. Then

$$
T(c, \lambda, p)=4|c|^{2-p} \int_{0}^{\left(1+\lambda^{-1}\right)^{\frac{1}{p}}}\left[1+\lambda-\lambda r^{p}\right]^{\frac{1-p}{p}} \mathrm{~d} r .
$$


Observe that with the change of variable $r=\left(1+\lambda^{-1}\right)^{\frac{1}{p}} S$ we see that

$$
\begin{aligned}
T(c, \lambda, p) & =4|c|^{2-p} \int_{0}^{1}\left(1+\lambda^{-1}\right)^{\frac{1}{p}}\left[(1+\lambda)\left(1-s^{p}\right)\right]^{\frac{1-p}{p}} \mathrm{~d} s \\
& =4|c|^{2-p} \lambda^{-\frac{1}{p}}(1+\lambda)^{\frac{2}{p}-1} \int_{0}^{1}\left(1-s^{p}\right)^{\frac{1-p}{p}} \mathrm{~d} s \\
& =4|c|^{2-p} \lambda^{-\frac{1}{p}}(1+\lambda)^{\frac{2}{p}-1} \frac{\Gamma\left(\frac{1}{p}\right)^{2}}{p \Gamma\left(\frac{2}{p}\right)}
\end{aligned}
$$

$T$ is increasing on $|c|$ if $p \in(1,2)$ and decreasing on $|c|$ if $p>2$ and independent of $|c|$ if $p=2$.

If we take $\lambda=1$,

$$
T(c, 1, p)=2^{\frac{2}{p}+1}|c|^{2-p} \frac{\Gamma\left(\frac{1}{p}\right)^{2}}{p \Gamma\left(\frac{2}{p}\right)}
$$

In particular, $T(c, 1,2)=2 \pi$ (independently of $c$ ).

We can also consider the dependence of $T$ on $\lambda$. We do this study for this particular example and in the following section we develop a general theory.

$$
\frac{\partial T}{\partial \lambda}(c, \lambda, p)=-\frac{4|c|^{2-p}}{p \lambda}\left(1+\frac{1}{\lambda}\right)^{\frac{1}{p}}(1+\lambda)^{\frac{1-2 p}{p}}(1+(p-1) \lambda) \int_{0}^{1}\left(1-s^{p}\right)^{\frac{1-p}{p}} \mathrm{~d} s<0
$$

Therefore the period $T$ is decreasing on $\lambda$.

Remark 2.5 If a continuous function $f$ satisfies $f(r t)=h(r) f(t)$, we can obtain the explicit expression of $f$. Let $c=f(1), g(t):=f(t) / f(1)$, and $\alpha=\ln g(e)$. Then $g(t s)=g(t) g(s)$. Also, for $t \neq 0,1=g(1)=g(t / t)=g(t) g(1 / t)$, and therefore $g\left(t^{-1}\right)=g(t)^{-1}$. If $n \in \mathbb{N}, g\left(t^{n}\right)=g(t)^{n}$, so, for $t \geq 0, g(t)=g\left(t^{\frac{n}{n}}\right)=g\left(t^{\frac{1}{n}}\right)^{n}$, and $g\left(t^{\frac{1}{n}}\right)=g(t)^{\frac{1}{n}}$. Hence, $g\left(t^{\frac{p}{q}}\right)=g(t)^{\frac{p}{q}}$ for every $p, q \in \mathbb{N}$, $q \neq 0$, and, by the density of $\mathbb{Q}$ in $\mathbb{R}$ and the continuity of $f, g\left(t^{r}\right)=g(t)^{r}$ for all $t \geq 0, r \in \mathbb{R}^{+}$.

Now, for $t>0, g(t)=g\left(e^{\ln t}\right)=g(e)^{\ln t}=e^{\ln g(e) \ln t}=t^{\ln g(e)}=t^{\alpha}$. Hence, $f(t)=\beta t^{\alpha}$ for $t \geq 0$. On the other hand, $1=g(1)=(g(-1))^{2}$, so $g(-1)= \pm 1$. Also, $f(-t)=g(-1) f(t)$ and thus, $f(-t)= \pm \beta t^{\alpha}$ for $t>0$. In summary,

$$
f(t)= \begin{cases}\beta t^{\alpha} & \text { if } t \geq 0 \\ \pm \beta(-t)^{\alpha} & \text { if } t<0\end{cases}
$$

If we further require $f$ to be injective, $f(t)=\beta|t|^{\alpha-1} t$, that is, $f$ is an $\alpha$-Laplacian.

\subsection{Dependence of $T$ on $\lambda$ and $c$}

Based on the approach used in Example 2.1, we study now the dependence of $T$ on $\lambda$ and $c$ in a general way. For simplicity, we will assume $c>0$. For the case $c<0$, just do the change of variable $y(t)=-x(t)$. 
We continue to assume the hypotheses for problem (13) and further assume that $f$ is a differentiable function. Let us divide the interval of integration in equation $(8)$ in $\left[F_{-}^{-1}((1+\right.$ $\left.\left.\left.\lambda^{-1}\right) F(c)\right), 0\right]$ and $\left[0, F_{+}^{-1}\left(\left(1+\lambda^{-1}\right) F(c)\right)\right]$. Observe that $F$ is injective restricted to any of the two intervals. For the nonnegative interval, taking the change of variables

$$
r=F_{+}^{-1}\left(\left(1+\lambda^{-1}\right) F(c s)\right)
$$

we see that

$$
\begin{aligned}
\int_{0}^{F_{+}^{-1}\left(\left(1+\lambda^{-1}\right) F(c)\right)}\left[\frac{1}{f\left(F_{+}^{-1}((1+\lambda) F(c)-\lambda F(r))\right)}-\frac{1}{f\left(F_{-}^{-1}((1+\lambda) F(c)-\lambda F(r))\right)}\right] \mathrm{d} r \\
=\int_{0}^{1}\left[\frac{1}{f\left(F_{+}^{-1}((1+\lambda)[F(c)-F(c s)])\right)}-\frac{1}{f\left(F_{-}^{-1}((1+\lambda)[F(c)-F(c s)])\right)}\right] \\
\quad \cdot \frac{\left[1+\lambda^{-1}\right] c f(c s)}{f\left(F_{+}^{-1}\left(\left(1+\lambda^{-1}\right) F(c s)\right)\right)} \mathrm{d} s .
\end{aligned}
$$

All the same, with the change of variables

$$
\begin{aligned}
& r=F_{-}^{-1}\left(\left(1+\lambda^{-1}\right) F(c s)\right), \\
& \int_{F_{-}^{-1}\left(\left(1+\lambda^{-1}\right) F(c)\right)}^{0}\left[\frac{1}{f\left(F_{+}^{-1}((1+\lambda) F(c)-\lambda F(r))\right)}-\frac{1}{f\left(F_{-}^{-1}((1+\lambda) F(c)-\lambda F(r))\right)}\right] \mathrm{d} r \\
& =\int_{1}^{0}\left[\frac{1}{f\left(F_{+}^{-1}((1+\lambda)[F(c)-F(c s)])\right)}-\frac{1}{f\left(F_{-}^{-1}((1+\lambda)[F(c)-F(c s)])\right)}\right] \\
& \quad \cdot \frac{\left[1+\lambda^{-1}\right] c f(c s)}{f\left(F_{-}^{-1}\left(\left(1+\lambda^{-1}\right) F(c s)\right)\right)} \mathrm{d} s .
\end{aligned}
$$

Now, for $\lambda \in \mathbb{R}^{+}$and $s \in[0,1]$, let

$$
\begin{aligned}
& \alpha(\lambda, s, c):=\left(1+\lambda^{-1}\right) c f(c s), \\
& \frac{\partial \alpha}{\partial \lambda}(\lambda, s, c)=-\lambda^{-2} c f(c s), \\
& \beta_{ \pm}(\lambda, s, c):=f\left(F_{ \pm}^{-1}\left(\left(1+\lambda^{-1}\right) F(c s)\right)\right), \\
& \frac{\partial \beta_{ \pm}}{\partial \lambda}(\lambda, s, c)=-\lambda^{-2} F(c s) \frac{f^{\prime}\left(F_{ \pm}^{-1}\left(\left(1+\lambda^{-1}\right) F(c s)\right)\right)}{f\left(F_{ \pm}^{-1}\left(\left(1+\lambda^{-1}\right) F(c s)\right)\right)} \\
& \gamma_{ \pm}(\lambda, s, c):=f\left(F_{ \pm}^{-1}((1+\lambda)[F(c)-F(c s)])\right), \\
& \frac{\partial \gamma_{ \pm}}{\partial \lambda}(\lambda, s, c)=[F(c)-F(c s)] \frac{f^{\prime}\left(F_{ \pm}^{-1}((1+\lambda)[F(c)-F(c s)])\right)}{f\left(F_{ \pm}^{-1}((1+\lambda)[F(c)-F(c s)])\right)} .
\end{aligned}
$$

Then

$$
T(\lambda, c)=\int_{0}^{1} \alpha(\lambda, s, c)\left[\frac{1}{\beta_{+}(\lambda, s, c)}-\frac{1}{\beta_{-}(\lambda, s, c)}\right]\left[\frac{1}{\gamma_{+}(\lambda, s, c)}-\frac{1}{\gamma_{-}(\lambda, s, c)}\right] \mathrm{d} s .
$$


Therefore,

$$
\begin{aligned}
\frac{\partial T}{\partial \lambda}(\lambda, c)= & \int_{0}^{1}\left\{\frac{\partial \alpha}{\partial \lambda}(\lambda, s, c)\left[\frac{1}{\beta_{+}(\lambda, s, c)}-\frac{1}{\beta_{-}(\lambda, s, c)}\right]\left[\frac{1}{\gamma_{+}(\lambda, s, c)}-\frac{1}{\gamma_{-}(\lambda, s, c)}\right]\right. \\
& +\alpha(\lambda, s, c)\left[\frac{\frac{\partial \beta_{-}}{\partial \lambda}(\lambda, s, c)}{\beta_{-}(\lambda, s, c)^{2}}-\frac{\frac{\partial \beta_{+}}{\partial \lambda}(\lambda, s, c)}{\beta_{+}(\lambda, s, c)^{2}}\right]\left[\frac{1}{\gamma_{+}(\lambda, s, c)}-\frac{1}{\gamma_{-}(\lambda, s, c)}\right] \\
& \left.+\alpha(\lambda, s, c)\left[\frac{1}{\beta_{+}(\lambda, s, c)}-\frac{1}{\beta_{-}(\lambda, s, c)}\right]\left[\frac{\frac{\partial \gamma_{-}}{\partial \lambda}(\lambda, s, c)}{\gamma_{-}(\lambda, s, c)^{2}}-\frac{\frac{\partial \gamma_{+}}{\partial \lambda}(\lambda, s, c)}{\gamma_{+}(\lambda, s, c)^{2}}\right]\right\} \mathrm{d} s .
\end{aligned}
$$

Observe that $\alpha,\left.f\right|_{[0,1]}, f^{\prime}, F, F_{+}^{-1}, \beta_{+}, \frac{\partial \beta_{-}}{\partial \lambda}, \gamma_{+}, \frac{\partial \gamma_{+}}{\partial \lambda}$ are nonnegative, while $\frac{\partial \alpha}{\partial \lambda}, F_{-}^{-1}, \beta_{-}, \frac{\partial \beta_{+}}{\partial \lambda}$, $\gamma_{-}, \frac{\partial \gamma_{-}}{\partial \lambda}$ are nonpositive. In general we cannot tell the sign of $T(\lambda, c)$ from this expression, but making certain assumptions we can simplify it to derive information.

Assume now $f$ is an odd function. Then $F_{-}^{-1}=-F_{+}^{-1}, \beta_{-}=-\beta_{+}$, and $\gamma_{-}=-\gamma_{+}$, so

$$
\begin{aligned}
\frac{\partial T}{\partial \lambda}(\lambda, c)= & 4 \int_{0}^{1} \frac{1}{\beta_{+}(\lambda, s, c) \gamma_{+}(\lambda, s, c)}\left[\frac{\partial \alpha}{\partial \lambda}(\lambda, s, c)\right. \\
& \left.-\alpha(\lambda, s, c)\left(\frac{\frac{\partial \beta_{+}}{\partial \lambda}(\lambda, s, c)}{\beta_{+}(\lambda, s, c)}+\frac{\frac{\partial \gamma_{+}}{\partial \lambda}(\lambda, s, c)}{\gamma_{+}(\lambda, s, c)}\right)\right] \mathrm{d} s .
\end{aligned}
$$

Now, if we differentiate equation (15) with respect to $c$,

$$
\begin{aligned}
\frac{\partial T}{\partial c}(\lambda, c)= & \int_{0}^{1}\left\{\frac{\partial \alpha}{\partial c}(\lambda, s, c)\left[\frac{1}{\beta_{+}(\lambda, s, c)}-\frac{1}{\beta_{-}(\lambda, s, c)}\right]\left[\frac{1}{\gamma_{+}(\lambda, s, c)}-\frac{1}{\gamma_{-}(\lambda, s, c)}\right]\right. \\
& +\alpha(\lambda, s, c)\left[\frac{\frac{\partial \beta_{-}}{\partial c}(\lambda, s, c)}{\beta_{-}(\lambda, s, c)^{2}}-\frac{\frac{\partial \beta_{+}}{\partial c}(\lambda, s, c)}{\beta_{+}(\lambda, s, c)^{2}}\right]\left[\frac{1}{\gamma_{+}(\lambda, s, c)}-\frac{1}{\gamma_{-}(\lambda, s, c)}\right] \\
& \left.+\alpha(\lambda, s, c)\left[\frac{1}{\beta_{+}(\lambda, s, c)}-\frac{1}{\beta_{-}(\lambda, s, c)}\right]\left[\frac{\frac{\partial \gamma_{-}}{\partial c}(\lambda, s, c)}{\gamma_{-}(\lambda, s, c)^{2}}-\frac{\frac{\partial \gamma_{+}}{\partial c}(\lambda, s, c)}{\gamma_{+}(\lambda, s, c)^{2}}\right]\right\} \mathrm{d} s .
\end{aligned}
$$

Observe that

$$
\begin{aligned}
& \frac{\partial \alpha}{\partial c}(\lambda, s, c)=\left(1+\lambda^{-1}\right)\left[f(c s)+c s f^{\prime}(c s)\right], \\
& \frac{\partial \beta_{ \pm}}{\partial c}(\lambda, s, c)=\left(1+\lambda^{-1}\right) s f(c s) \frac{f^{\prime}\left(F_{ \pm}^{-1}\left(\left(1+\lambda^{-1}\right) F(c s)\right)\right)}{f\left(F_{ \pm}^{-1}\left(\left(1+\lambda^{-1}\right) F(c s)\right)\right)} \\
& \frac{\partial \gamma_{ \pm}}{\partial c}(\lambda, s, c)=(1+\lambda)[f(c)-s f(c s)] \frac{f^{\prime}\left(F_{ \pm}^{-1}((1+\lambda)[F(c)-F(c s)])\right)}{f\left(F_{ \pm}^{-1}((1+\lambda)[F(c)-F(c s)])\right)} .
\end{aligned}
$$

Hence, $\frac{\partial \alpha}{\partial c}, \frac{\partial \beta_{+}}{\partial c}$ is positive and $\frac{\partial \beta_{-}}{\partial c}$ negative for $c \geq 0$. Assume now $f$ is an odd function.

$$
\begin{aligned}
\frac{\partial T}{\partial c}(\lambda, c)= & 4 \int_{0}^{1} \frac{1}{\beta_{+}(\lambda, s, c) \gamma_{+}(\lambda, s, c)}\left[\frac{\partial \alpha}{\partial c}(\lambda, s, c)\right. \\
& \left.-\alpha(\lambda, s, c)\left(\frac{\frac{\partial \beta_{+}}{\partial c}(\lambda, s, c)}{\beta_{+}(\lambda, s, c)}+\frac{\frac{\partial \gamma_{+}}{\partial c}(\lambda, s, c)}{\gamma_{+}(\lambda, s, c)}\right)\right] \mathrm{d} s .
\end{aligned}
$$




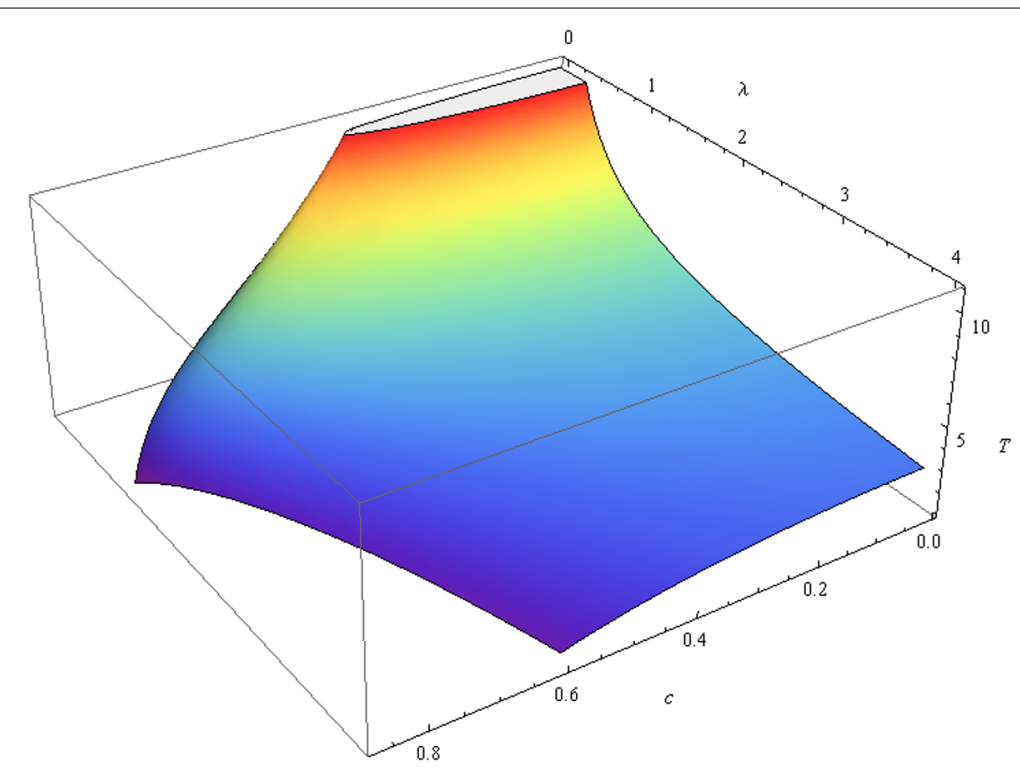

Figure 1 Graph of the period $T$ function of $c$ and $\lambda$.

Example 2.2 Let $f:(-1,1) \rightarrow \mathbb{R}, f(x):=x / \sqrt{1-x^{2}}, x \in \mathbb{R}$, and consider problem (13). ${ }^{\mathrm{a}}$ Then

$$
F(x)=1-\sqrt{1-x^{2}}, \quad F_{+}^{-1}(x)=\sqrt{2 x-x^{2}} .
$$

In order for the conditions in Corollary 2.3 to be satisfied we need

$$
(1+\lambda) F(c)<1, \quad\left(1+\lambda^{-1}\right) F(c)<1,
$$

that is,

$$
|c|<\min \left\{\frac{\sqrt{\lambda(\lambda+2)}}{\lambda+1}, \frac{\sqrt{2 \lambda+1}}{\lambda+1}\right\} .
$$

In Figure 1 we plot how the period varies as a function of $c$ and $\lambda$. Observe how the period is decreasing in both parameters and $\lim _{c, \lambda \rightarrow 0} T(\lambda, c)=+\infty$.

Example 2.3 Let $f$ be the bounded $\phi$-Laplacian [32] given by $f: \mathbb{R} \rightarrow(-1,1), f(x):=$ $x / \sqrt{1+x^{2}}, x \in \mathbb{R}$, and consider problem (13). $f$ is effectively the inverse function of the one in the previous example. Then

$$
F(x)=\sqrt{1+x^{2}}-1, \quad F_{+}^{-1}(x)=\sqrt{2 x+x^{2}} .
$$

The conditions in Corollary 2.3 are satisfied without any further restrictions. In Figure 2 we plot how the period varies as a function of $c$ and $\lambda$. Observe in this plot how the period is decreasing in $\lambda$, increasing in $c$ and $\lim _{\lambda \rightarrow 0} T(c, \lambda)=\lim _{c \rightarrow+\infty} T(c, \lambda)=+\infty$. 


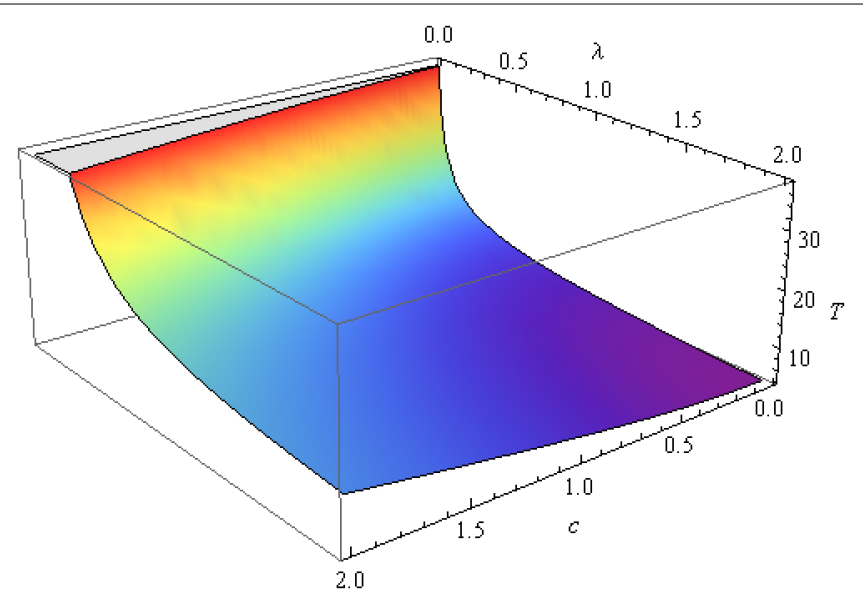

Figure 2 Graph of the period $T$ function of $c$ and $\lambda$.

\section{Problems with reflection}

Let us consider again the problem that motivated this paper in the Introduction, obtaining solutions of problem (3) in the case $\varphi(t)=-t$. Hence, consider all of the problems (1)-(5) in the case $\varphi(t)=-t$.

Observe that Lemma 1.1 (following Remark 1.1) can be trivially extended to the following lemma.

Lemma 3.1 Letf $:\left(\tau_{1}, \tau_{2}\right) \rightarrow\left(\sigma_{1}, \sigma_{2}\right)$ a locally Lipschitz a.c. function with a.c. inverse. Then $x$ is a solution of the first order differential equation with involution (3) if and only if $x$ is a solution of the second order ordinary differential equation (4).

As was shown in Section 1, problem (4) is equivalent to problem (5). We can now state the following corollary of Theorem 2.2 regarding the periodicity of problem (3) as foreseen in Section 1.

Corollary 3.2 Let $f:\left(\tau_{1}, \tau_{2}\right) \rightarrow\left(\sigma_{1}, \sigma_{2}\right)$ an increasing locally Lipschitz a.c. function with a.c. inverse such that $0 \in\left(\tau_{1}, \tau_{2}\right), f(0)=0$, and $c>0$. Assume $2 F(c)<\min \left\{F\left(\tau_{1}\right), F\left(\tau_{2}\right)\right\}$. Then, if $x_{c}(t)$ is a solution of problem (6) and we assume there exist $\bar{c}_{1}, \bar{c}_{2} \in \mathbb{R}, \bar{c}_{1}<\bar{c}_{2}$, such that $2 \max \left\{F\left(\bar{c}_{1}\right), F\left(\bar{c}_{2}\right)\right\}<\min \left\{F\left(\tau_{1}\right), F\left(\tau_{2}\right)\right\}$ and $\left(x_{\bar{c}_{1}}(b)-\bar{c}_{1}\right)\left(x_{\bar{c}_{2}}(b)-\bar{c}_{2}\right)<0$, then problem (3) must have at least a solution.

We now give an example in which there is no need to find $\bar{c}_{1}, \bar{c}_{2} \in \mathbb{R}$ under the conditions of Corollary 3.2 because the function determining the period has a simple inverse.

Example 3.1 Take again $f(t):=|t|^{p-2} t, p>1, c>0$, and consider the problem

$$
x^{\prime}(t)=|x(-t)|^{p-2} x(-t), \quad t \in \mathbb{R}, \quad x(0)=c .
$$

By Corollaries 2.3 and 3.2 and Example 2.1, we see that the solutions of are periodic for every $c \neq 0$ and

$$
T(c, 1, p)=2^{\frac{2}{p}+1} c^{2-p} \frac{\Gamma\left(\frac{1}{p}\right)^{2}}{p \Gamma\left(\frac{2}{p}\right)} .
$$


Consider now the problem

$$
x^{\prime}(t)=|x(-t)|^{p-2} x(-t), \quad t \in \mathbb{R}, \quad x(a)=x(b) .
$$

There is a unique solution for problem (17) for $p \in(2,+\infty)$. Just take the unique solution of problem (16) with

$$
c=\left(\frac{b-a}{2^{\frac{2}{p}+1}} p \frac{\Gamma\left(\frac{2}{p}\right)}{\Gamma\left(\frac{1}{p}\right)^{2}}\right)^{\frac{1}{2-p}} .
$$

Observe that for $p \in(0,2)$ the function $f$ is not locally Lipschitz, and therefore we cannot apply Lemma 3.1 .

\section{Competing interests}

The authors declare that they have no competing interests.

\section{Authors' contributions}

All authors contributed equally to the writing of this paper. All authors read and approved the final manuscript.

\section{Acknowledgements}

The work was partially supported by FEDER and Ministerio de Economía y Competitividad, Spain, project MTM2013-43014-P. The second author was supported by FPU scholarship, Ministerio de Educación, Cultura y Deporte, Spain and Xunta de Galicia (Spain), project EM2014/032.

\section{Endnote}

a The diffeomorphisms $f$ in this example has been widely studied by Bereanu and Mawhin (see, for instance, [32, 33]) and is a type of singular $\phi$-Laplacian known as the mean curvature operator of the Minkowski space. Its inverse, the mean curvature operator of the Euclidean space, also studied in [32], appears in Example 2.3.

Received: 11 November 2015 Accepted: 16 February 2016 Published online: 29 February 2016

\section{References}

1. Cabada, A, Tojo, FAF: Comparison results for first order linear operators with reflection and periodic boundary value conditions. Nonlinear Anal. 78, 32-46 (2013)

2. McShane, EJ: Integration. Princeton University Press, Princeton (1967)

3. Stromberg, KR: An Introduction to Classical Real Analysis. Chapman \& Hall, London (1996)

4. Cabada, A, Tojo, FAF: Existence results for a linear equation with reflection, non-constant coefficient and periodic boundary conditions. J. Math. Anal. Appl. 412(1), 529-546 (2014)

5. Cabada, A, Tojo, FAF: Solutions of the first order linear equation with reflection and general linear conditions. Global Journal of Mathematical Sciences 2(1), 1-8 (2013)

6. Drábek, P: On the variational eigenvalues which are not of Ljusternik-Schnirelmann type. Abstr. Applied Analysis 2012, Article ID 434631 (2012)

7. Binding, P, Drábek, P, Huang, Y: The range of the $p$-Laplacian. Applied Mathematics Letters 10(6), 77-82 (1997)

8. Drábek, P, Manásevich, R, et al.: On the closed solution to some nonhomogeneous eigenvalue problems with p-Laplacian. Differential Integral Equations 12(6), 773-788 (1999)

9. Drábek, P, Girg, P, Manásevich, R: Generic Fredholm alternative-type results for the one dimensional $p$-Laplacian. NoDEA Nonlinear Differential Equations Appl. 8(3), 285-298 (2001)

10. del Pino, M, Drábek, $P$, Manásevich, R: The Fredholm alternative at the first eigenvalue for the one dimensional p-Laplacian. J. Differential Equations 151(2), 386-419 (1999)

11. Drábek, P, García-Huidobro, M, Manásevich, R: Positive solutions for a class of equations with a $p$-Laplace like operator and weights. Nonlinear Anal. 71(3), 1281-1300 (2009)

12. Sanchez, J, Vergara, V: Exact number of solutions for a Neumann problem involving the $p$-Laplacian. Electron. J. Differential Equations 2014(30), 1-10 (2014)

13. Del Pino, M, Elgueta, M, Manásevich, R: A homotopic deformation along $p$ of a Leray-Schauder degree result and existence for $\left(\left|u^{\prime}\right|^{p-2} u^{\prime}\right)^{\prime}+f(t, u)=0, u(0)=u(T)=0, p>1$. J. Differential Equations 80(1), 1-13 (1989)

14. Cabada, A, Cid, JÁ, Tvrdỳ, M: A generalized anti-maximum principle for the periodic one-dimensional $p$-Laplacian with sign-changing potential. Nonlinear Anal. 72(7), 3436-3446 (2010)

15. Cabada, A, Lomtatidze, A, Tvrdy, M: Periodic problem involving quasilinear differential operator and weak singularity. Adv. Nonlinear Stud. 7(4), 629 (2007)

16. Chvátal, M, Došlý, O: Variational method and conjugacy criteria for half-linear differential equations. Arch. Math. (Brno) 49, 9-16 (2013)

17. Bognár, G, Došly, $\mathrm{O}$ : The ratio of eigenvalues of the Dirichlet eigenvalue problem for equations with one-dimensional-Laplacian. Abstr. Applied Analysis 2010, Article ID 123975 (2010)

18. Došlỳ, O, Özbekler, A, Hilscher, RŠ: Oscillation criterion for half-linear differential equations with periodic coefficients. J. Math. Anal. Appl. 393(2), 360-366 (2012) 
19. Heikkilä, S, Seikkala, S: On singular, functional, nonsmooth and implicit phi-Laplacian initial and boundary value problems. J. Math. Anal. Appl. 308(2), 513-531 (2005)

20. O'Regan, D: Existence results for differential equations with reflection of the argument. J. Aust. Math. Soc. 57(2), 237-260 (1994)

21. Karakostas, GL: Solvability of the $\Phi$-Laplacian with nonlocal boundary conditions. Appl. Math. Comput. 215, 514-523 (2009)

22. Cabada, A, Staněk, S: Functional fractional boundary value problems with singular $\varphi$-Laplacian. Appl. Math. Comput. 219(4), 1383-1390 (2012)

23. Agarwal, RP, O'Regan, D: An Introduction to Ordinary Differential Equations. Springer, New York (2008)

24. Cecchi, M, Došlá, Z, Marini, M: Oscillation of a class of differential equations with generalized phi-Laplacian. Proc. Roy. Soc. Edinburgh 143A, 493-506 (2013)

25. Liu, CS: Developing an SL(2, R) Lie-group shooting method for a singular $\varphi$-Laplacian in a nonlinear ODE. Commun. Nonlinear Sci. Numer. Simul. 18(9), 2327-2339 (2013)

26. Klén, R, Vuorinen, M, Zhang, X: Inequalities for the generalized trigonometric and hyperbolic functions. J. Math. Anal. Appl. 409(1), 521-529 (2014)

27. Busenberg, SN, Travis, CC: On the use of reducible-functional differential equations in biological models. J. Math. Anal. Appl. 89(1), 46-66 (1982)

28. Edmunds, DE, Gurka, P, Lang, J: Properties of generalized trigonometric functions. J. Approx. Theory 164(1), 47-56 (2012)

29. Bhayo, BA, Vuorinen, M: On generalized trigonometric functions with two parameters. J. Approx. Theory 164(10), 1415-1426 (2012)

30. Jiang, WD, Wang, MK, Chu, YM, Jiang, YP, Qi, F: Convexity of the generalized sine function and the generalized hyperbolic sine function. J. Approx. Theory 174, 1-9 (2013)

31. Takeuchi, S: Generalized Jacobian elliptic functions and their application to bifurcation problems associated with p-Laplacian. J. Math. Anal. Appl. 385(1), 24-35 (2012)

32. Bereanu, C, Jebelean, P, Mawhin, J: Radial solutions for some nonlinear problems involving mean curvature operators in Euclidean and Minkowski spaces. P. Am. Math. Soc. 137(1), 161-169 (2009)

33. Bereanu, C, Jebelean, P, Mawhin, J: Radial solutions for Neumann problems involving mean curvature operators in Euclidean and Minkowski spaces. Math. Nachr. 283(3), 379-391 (2010)

\section{Submit your manuscript to a SpringerOpen ${ }^{\circ}$ journal and benefit from:}

- Convenient online submission

- Rigorous peer review

Immediate publication on acceptance

- Open access: articles freely available online

- High visibility within the field

Retaining the copyright to your article 